\title{
Phase separation in polyaniline with near-field scanning optical microscopy
}

\author{
Pei-Kuen Wei, Jui-Hung Hsu, Wunshain Fann, Kuen-Ru Chuang, Hsun-Tsing Lee, \\ and Show-An Chen
}

\begin{abstract}
We report the studies of conjugated polymers, polyaniline thin films, with a near-field scanning optical microscope. Because of the absorption variation in different oxidation states, transmission-mode nearfield scanning optical microscope images were employed to map out the distribution of the oxidation states on a submicrometer scale. When the near-field wavelength is varied (between 632.8 and 543.5 $\mathrm{nm}$ ), the phase separation between the oxidized and the reduced repeated units, with domain sizes on a nanometer-length scale, is observed. (C) 1997 Optical Society of America
\end{abstract}

The near-field scanning optical microscope ${ }^{1-4}$ (NSOM) is a novel optical instrument that combines the high spatial resolution of a scanning probe microscope and the advantages of a traditional optical microscope; it is nondestructive and its contrast mechanism is versatile. The unique capability of imaging beyond the diffraction limit makes the NSOM a powerful tool for investigating the optical properties on a nanometer scale. In addition, because a NSOM can be operated at several optical wavelengths, it is useful in spectroscopic analysis. The combination of spectroscopy with near-field optics opens up new possibilities in submicrometer sciences and technology. In this paper, we report the NSOM studies of a conjugated polymer, polyaniline (PAn). When the excitation wavelength is tuned, the inhomogeneity in the polymer sample is observed on a submicrometer-length scale.

PAn is an environmentally stable conducting polymer with high conductivity of the order of 10 $\mathrm{S} / \mathrm{cm}$ after doping, and it possesses the electrical properties of metals. Nevertheless, the mechanical properties and processibility commonly associated with the polymers are still maintained. ${ }^{5}$ The PAn base consists of equal numbers of re-

P. K. Wei, J. H. Hsu, and W. S. Fann are with the Institute of Atomic and Molecular Sciences, Academia Sinica and Department of Physics, National Taiwan University, P. O. Box 23-166, Taipei, Taiwan. K. R. Chuang, H. T. Lee, and S. A. Chen are with the Department of Chemical Engineering, National Tsing Hwa University, Hsinchu, Taiwan.

Received 20 August 1996; revised manuscript received 2 December 1996.

0003-6935/97/153301-04\$10.00/0

(C) 1997 Optical Society of America duced $\left[-\left(\mathrm{C}_{6} \mathrm{H}_{4}\right) \mathrm{NH}\left(\mathrm{C}_{6} \mathrm{H}_{4}\right) \mathrm{NH}-\right]$ and oxidized $\left[-\left(\mathrm{C}_{6} \mathrm{H}_{4}\right) \mathrm{N}=\left(\mathrm{C}_{6} \mathrm{H}_{4}\right)=\mathrm{N}-\right]$ repeat units. It is formed by the chemical or electrochemical oxidative polymerization of aniline. Before doping, the PAn in neutral form can be prepared in three different oxidation states: leucoemeraldine, emeraldine, and pernigraniline. The absorption spectra of these three states are shown in Fig. 1. The leucoemeraldine state is in reduced form and is transparent in the visible light range. The emeraldine oxidation state is half oxidized and has a maximum absorption in the visible range. From the leucoemeraldine to the emeraldine state, the absorption in the visible range increases and the peak is at $630 \mathrm{~nm}$. The pernigraniline state is a fully oxidized form. From the emeraldine to the pernigraniline state, the absorption in the visible range is reduced and the absorption peak shifts to the green light range. To produce the highly conducting PAn by protonic acid doping, the emeraldine state is necessary. However, it consists of two types of repeated units, which could phase separate in the solid film; this is of fundamental importance in understanding whether phase separation occurs. Because of the different absorption spectra associated with the different oxidation states, the NSOM can be employed to image the phase separation on a nanometer-length scale. In addition, it is clear from Fig. 1 that if two wavelengths, 632.8 and $543.5 \mathrm{~nm}$, are used, the two-color NSOM images will have nearly the same contrast when the area consists mainly of reduced repeated units. On the other hand, if the region consists mainly of oxidized repeat units, these two images will give the opposite contrast. Therefore the two-color NSOM provides a method for distinguishing the local distribution of oxidation states of PAn. 


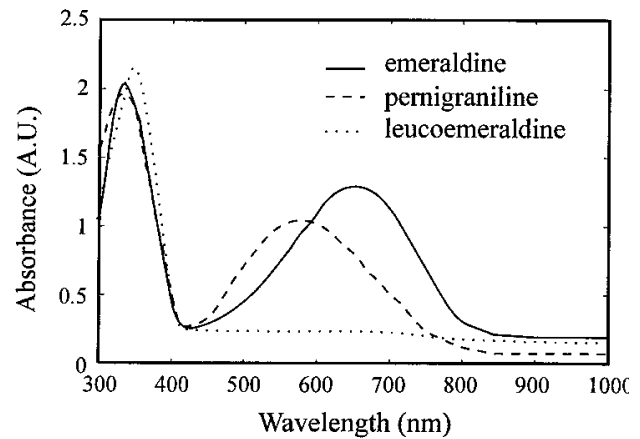

Fig. 1. Optical absorption spectrum of PAn in three different oxidation states: leucoemeraldine, emeraldine, and pernigraniline.

The schematic of the dual-color NSOM used in this experiment is shown in Fig. 2. In the heart of the NSOM is the metal-coated tapered fiber probe. ${ }^{6}$ The tapered fiber has a tip size of $100 \mathrm{~nm}$ and is coated with 75-nm-thick A1. The input light is from either a $2-\mathrm{mW}$ red $\mathrm{He}-\mathrm{Ne}$ laser $(632.8 \mathrm{~nm})$ or a $0.5-\mathrm{mW}$ green He-Ne laser $(543.5 \mathrm{~nm})$. These lasers are coupled into the tapered fiber probe to serve as the nearfield optical sources. Another key element in the NSOM is the tip-sample distance regulation. We used the shear-force feedback technique to maintain the tip and sample separation within $10 \mathrm{~nm} \cdot .^{7,8}$ In the shear-force technique, the probe was fixed on a dither piezoelectric transducer (PZT) $[0.02$ in. thick $\times$ 0.2 in. wide $\times 0.5 \mathrm{in}$. long $(0.05 \mathrm{~cm}$ thick $\times 0.5 \mathrm{~cm}$ wide $\times 1.27 \mathrm{~cm}$ long)] and set into vibration near its resonance. The resonant frequency was $\sim 95 \mathrm{kHz}$, and the driven voltage was $0.01 \mathrm{~V}$ peak to peak. A 5 -mW red He-Ne laser was then focused onto the tip from the side, and the scattered light was detected with a photodiode and a lock-in amplifier. The measured ac amplitude in the lock-in amplifier was proportional to the tip's vibrational amplitude. As the tip approached the sample, the amplitude decreased because of the interaction force between the tip and the sample surface. This shear-force signal was

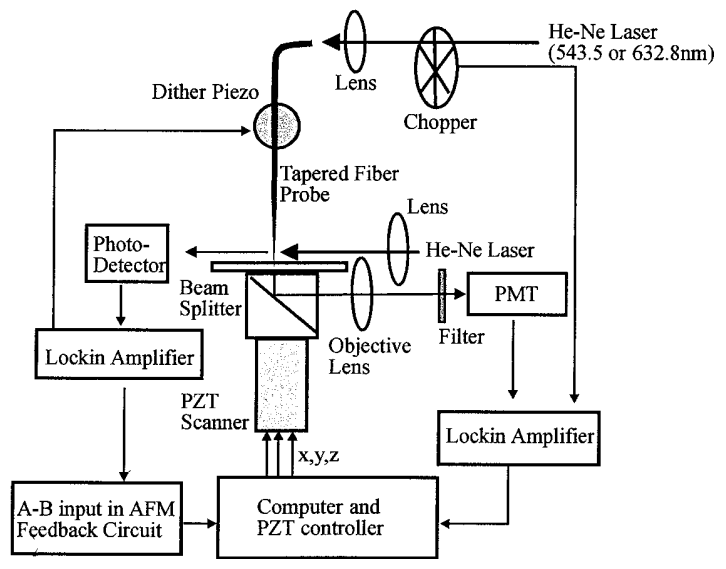

Fig. 2. Schematic of the transmission-mode NSOM: PMT, photomultiplier tube PZT, piezoelectric transducer.

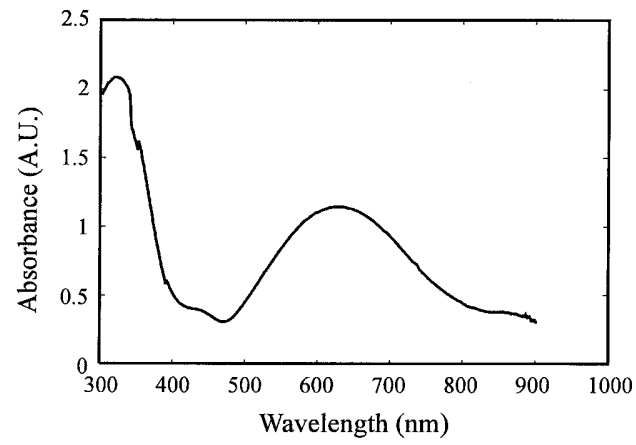

Fig. 3. Absorption spectrum of the PAn sample used in the experiment.

sent to the PZT scanning stage to regulate the tipsample distance. The feedback signal generated a topographic image, which was important for interpreting the optical image. In shear-force feedback, the undamped vibrational amplitude is estimated to be $\sim 12 \mathrm{~nm}$ by a modified beam-diffraction method. ${ }^{8}$ For vibrational amplitude detection, the noise level at the $1-\mathrm{kHz}$ bandwidth is $\sim 0.33 \mathrm{~nm}$, which corresponds to a signal-to-noise ratio of 36 . The shear-force decay length is $\sim 10 \mathrm{~nm}$. Hence the vertical distance resolution in shear-force images is $0.28 \mathrm{~nm}$. For recording a transmission-mode optical image, a mirror was used to couple the light out of the NSOM. The mirror was placed directly below the sample and mounted at $45^{\circ}$ on the PZT scanning stage. The optical signal traveling through the sample was reflected at a right angle by the mirror. Subsequently a long working distance objective was used to collect the light to a photomultiplier tube. The signal from the photomultiplier tube was sent to a lock-in amplifier. The signal-to-noise ratio of this transmissionmode NSOM is $\sim 100: 1$, which ensures its capability of distinguishing the small absorption variation in the PAn sample.

Our experiments were performed on 1-methyl-2pyrrolidone (NMP)-plasticized-PAn film. It is prepared with the PAn $\mathrm{HCl}$ powder. The powder synthesized in aqueous $\mathrm{HCl}$ is insoluble in common organic solvents and even in 1-methyl-2-pyrrolidone, which is the only organic solvent found so far that can dissolve high-molecular-weight PAn. However, when $\mathrm{PAn} \mathrm{HCl}$ is treated with aqueous $\mathrm{NH}_{4} \mathrm{OH}$, the resulting PAn base is soluble in NMP and can be cast into a flexible film with residual NMP as the plasticizer. During film casting, NMP is difficult to remove completely. The resulting PAn film usually contains a considerable amount of NMP, 10\%-18\% by weight and is termed NMP-plasticized PAn (abbreviated to NMP-p-PAn) film. The orientation of the polymer chains on the sample is completely random. The various properties of NMP-p-PAn film are discussed elsewhere. ${ }^{9}$

The NMP-p-PAn film had a thickness of $80 \mathrm{~nm}$ and was coated on a glass substrate. Figure 3 shows the absorption spectrum of the sample. Compared with 


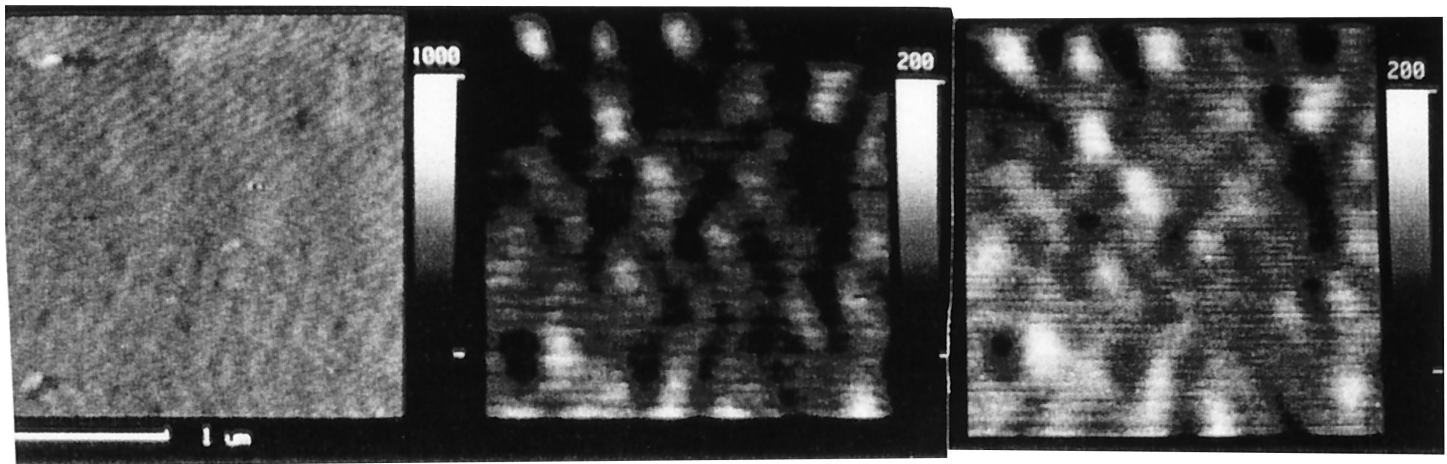

(a)

(b)

(c)

Fig. 4. (a) Topographic image, (b) optical image taken with a 632.8-nm light source, (c) optical image taken with a 543.5-nm light source. The optical images with different wavelengths have the same contrast. The scan area is $2.6 \mu \mathrm{m} \times 2.6 \mu \mathrm{m}$. The maximum vertical scale in (a) is $100 \mathrm{~nm}$.

Fig. 1, the sample is just about half oxidized, similar to the emeraldine state. However, this is the macroscopic average spectrum, which does not reflect the underlying details of the oxidation-state properties. When a NSOM is used, information on the oxidation states on a 100-nm scale can be distinguished. Figure 4(a) shows the topographic image, and Figs. 4(b) and 4(c) show the optical images obtained with 632.8- and 543.5-nm light, respectively. The topographic image is quite smooth; however, the optical images show the inhomogeneity on a the submicrometer scale. Because this is a transmission image, the bright region represents the area where the absorption is smaller. In the bright region the degree of oxidation is less than that in the dark area. In addition, the images of the two different wavelengths show the same contrast. This indicates that this area consists mainly of reduced repeated units. Figure 5 shows the images taken at a different area of the same sample. Figure 5(a) is the topographic image, and Figs. 5(b) and 5(c) show the optical images taken at 632.8 and $543.5 \mathrm{~nm}$, respectively. Again this image also indicates a nonuniform distribution of oxidation levels. However, the two optical images exhibit opposite contrast, that is, the brighter area in the 632.8-nm image is the darker area in the 543.5-nm image. Hence it is suggested that this area consists of domains of oxidized repeated units. The full width at half-maximum (FWHM) of the smallest resolved feature in the absorption image, Fig. 5(c), is $\sim 120 \mathrm{~nm}$. Note that the domains in Fig. 5 are larger than the film thickness, $80 \mathrm{~nm}$. Hence the sample should not have a region that allows for the full overlap of different phases.

It is interesting to compare the images obtained by the NSOM technique with those obtained by means of other microscopies. The scanning electron microscopy image of the NMP-p-PAn film exhibits a smooth and featureless surface morphology and, after extraction with tetrahydrofuran, a fibrilar morphology. ${ }^{10}$ The atomic force microscopy image of the film coated on a highly ordered pyrolytic graphite substrate shows aggregates of small globules with a diameter of $\sim 50 \mathrm{~nm} .{ }^{11}$ Both scanning electron and atomic force microscopy images reveal only the roughness of the film surface and provide no information on oxidation levels. Not only can the NSOM provide an image of the surface variation, but it can also map out the

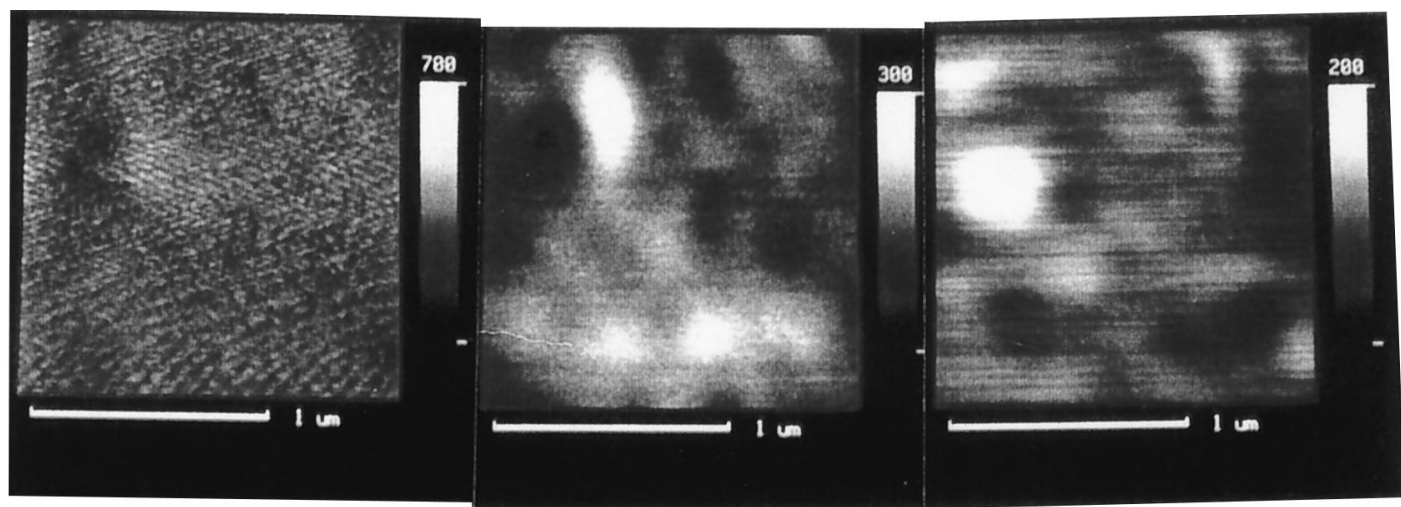

(a)

(b)

(c)

Fig. 5. (a) Topographic image, (b) optical image taken with a 632.8-nm light source, (c) optical image taken with a 543.5-nm light source. Note that a certain area in the two wavelength optical images has opposite contrast. The scan area is $1.6 \mu \mathrm{m} \times 1.5 \mu \mathrm{m}$. The maximum vertical scale in (a) is $70 \mathrm{~nm}$. 
optical absorption differences. Hence it is a powerful tool for imaging oxidation variations with super resolution. In general, it should be a valuable instrument for studying the phase-separation phenomena that commonly occurs in polymer blends.

In summary, we have studied PAn by using a twocolor NSOM. The absorption images exhibit contrast that arises from the absorption changes in the different oxidation states of PAn. When red and green light sources are used, a phase separation between the oxidized and the reduced repeated units is observed. The NSOM provides a method for probing inhomogeneity, in particular phase separation, in conjugated polymers on a nanometer scale.

This research was funded by the National Science Council, Taiwan.

\section{References}

1. E. Betzig and J. K. Trautman, "Near-field optics: microscopy, spectroscopy and surface modification beyond the diffraction limit," Science 257, 189-195 (1992).

2. U. Ch. Fischer, U. T. Durig, and D. W. Pohl, "Near-field optical scanning microscopy in reflection,” Appl. Phys. Lett. 42, 249251 (1988).

3. R. Toledo-Crow, P. C. Yang, Y. Chen, and M. Vaez-Iravani,
"Near-field differential scanning optical microscope with atomic force regulation," Appl. Phys. Lett. 60, 2957-2959 (1992).

4. W. P. Ambrose, P. M. Goodwin, J. C. Martin, and R. A. Keller, "Single molecule detection and photochemistry on a surface using near-field optical excitation," Phys. Rev. Lett. 72, 160 163 (1994).

5. A. G. MacDiarmid, "The polyanilines: a novel class of conducting polymers," in Proceedings of the 1991 Nobel Symposium on Conjugated Polymers and Related Materials, W. R. Salaneck, ed. (Oxford U. Press, New York, 1993), pp. 73-98.

6. E. Betzig, J. K. Trautman, T. D. Harris, J. S. Weiner, and R. L. Kostelak, "Breaking the diffraction barrier: optical microscopy on a nanometric scale," Science 251, 1468-1470 (1991).

7. E. Betzig, P. L. Finn, and J. S. Weiner, "Combined shear force and near-field scanning optical microscopy," Appl. Phys. Lett. 60, 2484-2486 (1992).

8. C. C. Wei, P. K. Wei, and W. S. Fann, "Direct measurements of true vibrational amplitude in shear force microscopy," Appl. Phys. Lett. 67, 3835-3837 (1995).

9. H. T. Lee, K. R. Chuang, S. A. Chen, P. K. Wei, J. H. Hsu, and W. S. Fann, "Conductivity relaxation of 1-methyl-2pyrrolidone-plasticized polyaniline film," Macromolecules 28, 7645-7652 (1995).

10. S. A. Chen and H. T. Lee, "Polyaniline plasticized with 1-methyl-2-pyrrolidone: structure and doping behavior," Macromolecules 26, 3254-3261 (1993).

11. S. A. Chen and H. T. Lee, "Structure and Properties of poly(acrylic acid)-doped polyaniline," Macromolecules 28, 2858-2866 (1995). 